\title{
Role of Vancomycin Minimum Inhibitory Concentrations by Modified Population Analysis Profile Method and Clinical Outcomes in High Inoculum Methicillin-Resistant Staphylococcus aureus Infections
}

\author{
Trang D. Trinh · Evan J. Zasowski · Kimberly C. Claeys · Anthony M. Casapao • \\ Matthew Compton · Abdalhamid Lagnf · Shravya D. Kidambi · \\ Donald P. Levine · Michael J. Rybak \\ Received: December 18, 2017 / Published online: February 22, 2018 \\ (c) The Author(s) 2018. This article is an open access publication
}

\begin{abstract}
Introduction: Vancomycin remains the standard of care for invasive methicillin-resistant Staphylococcus aureus (MRSA) infections. Treatment failures from heteroresistant vancomycinintermediate subpopulations (hVISA) are challenging to detect. Minimum inhibitory concentrations (MIC) identified by modified population analysis profile (PAP) is an alternative testing method. The aim of this study was to evaluate the role of PAP MIC on vancomycin failures in two high inoculum infections: MRSA infective endocarditis and pneumonia.
\end{abstract}

Enhanced content To view enhanced content for this article go to https://doi.org/10.6084/m9.figshare. 5813550 .

T. D. Trinh · E. J. Zasowski · M. Compton · A. Lagnf · S. D. Kidambi · M. J. Rybak $(\bowtie)$ Anti-Infective Research Laboratory, Department of Pharmacy Practice, Eugene Applebaum College of Pharmacy and Health Sciences, Wayne State University, Detroit, MI, USA

e-mail: m.rybak@wayne.edu

T. D. Trinh

Department of Clinical Pharmacy, School of Pharmacy, University of California, San Francisco, CA, USA

E. J. Zasowski

Department of Pharmacy Practice and Translational Research, College of Pharmacy, University of

Houston, Houston, TX, USA
Methods: Retrospective, observational study at Detroit Medical Center from 2008 to 2016. Adults $\geq 18$ years with $\geq 1$ positive MRSA blood culture from IE or pneumonia source and received $\geq 48 \mathrm{~h}$ vancomycin were included. The primary outcome was composite failure: MRSA bacteremia $\geq 7$ days or 30-day all-cause mortality.

Results: A total of 191 patients were included; 47.6\% IE and 52.4\% pneumonia. About 19\% were hVISA isolates, median vancomycin PAP MIC of $3(2,3)$. More than half $(54.5 \%)$ experienced composite failure with a larger proportion of PAP MIC $\geq 4 \mathrm{mg} / \mathrm{L}$ in this group ( $25 \mathrm{vs}$. $15 \%, p=0.086)$. Patients with IE experienced prolonged bacteremia whereas patients with pneumonia experienced higher 30-day

\section{K. C. Claeys}

Department of Pharmacy Practice, School of

Pharmacy, University of Maryland, Baltimore, MD, USA

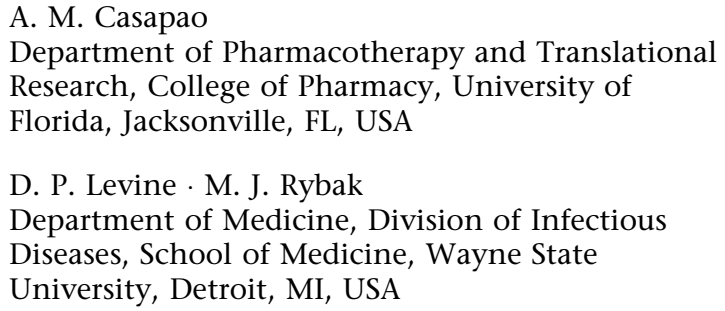


mortality. On logistic regression analysis, age [adjusted odds ratio (aOR), 1.026; 95\% confidence interval (CI), 1.005-1.047; $p=0.014]$ and APACHE II score (aOR 1.039; 95\% CI, $1.004-1.076 ; p=0.029$ ) independently predicted composite failure.

Conclusion: Vancomycin PAP MIC may be a more relevant predictor of patient outcomes in persistent bacteremic MRSA infections (e.g., IE). This susceptibility method is less applicable in other high inoculum infections with shorter bacteremia durations and higher mortality rates (e.g., pneumonia).

Keywords: Clinical outcomes; hVISA; Infective endocarditis; Pneumonia

\section{INTRODUCTION}

Methicillin-resistant Staphylococcus aureus (MRSA) infections with high bacterial burden (e.g., infective endocarditis and pneumonia) are difficult to treat and contribute to substantial morbidity and mortality $[1,2]$. These resistant infections result in prolonged, complicated, and costly hospitalizations in the United States [2]. Infective endocarditis and pneumonia resulted in more than 50,000 deaths with associated medical costs exceeding US\$10 billion [3, 4]. Vancomycin remains the recommended antibiotic for these invasive MRSA infections [5]. However, reduced vancomycin susceptibility phenotypes in high inoculum infections further complicate treatment success.

Heterogeneous vancomycin-intermediate $S$. aureus (hVISA) phenotypes include subpopulations that exhibit higher vancomycin minimum inhibitory concentrations (MIC) than reported by standard broth microdilution (BMD) methods [6]. These phenotypes are less susceptible to vancomycin and have the potential to become vancomycin-intermediate $S$. aureus (VISA) during prolonged or sub-therapeutic treatment with vancomycin. The isolates are defined by a population analysis profile (PAP) with an area under the curve ratio between $\geq 0.9$ and $<1.3$ when compared to the curve for the Mu3 control isolate [7]. This testing method is more costly and labor-intensive and thus not routinely performed in clinical microbiology laboratories.

However, it has been reported that hVISA proportions are higher among MRSA isolates with a susceptible vancomycin Etest MIC of $2 \mathrm{mg} / \mathrm{L}$ [8]. Among MRSA IE isolates, hVISA prevalence ranges between 19 and 34\% with an association between hVISA and higher vancomycin PAP MIC [9]. Owing to contemporary susceptibility testing methods, it is not currently feasible to routinely test for hVISA in the clinical setting. This setback thereby complicates successful management of invasive MRSA infections that necessitate bactericidal activity with detailed susceptibility results (i.e., detection of less susceptible subpopulations). The clinical application of a modified susceptibility method to predict vancomycin treatment response may provide a compromise between cumbersome hVISA testing methods and meaningful susceptibility results. In a study of 202 MRSA infective endocarditis patients, vancomycin PAP MIC $\geq 4 \mathrm{mg} / \mathrm{L}$ was associated with treatment failure, defined as persistent bacteremia for $\geq 7$ days or MRSA-attributable mortality [10].

On the basis of higher hVISA prevalence within infective endocarditis, it was hypothesized that heteroresistant subpopulations are also likely to exist within other high burden infections (e.g., $10^{8}-10^{10} \mathrm{cfu} / \mathrm{mL}$ bacteria). Pneumonia serves as a dense bacterial source [11]; however, the role of vancomycin PAP MIC to predict outcomes in MRSA pneumonia remains to be determined.

\section{METHODS}

A retrospective, observational study of patients admitted between January 1, 2008 and June 30, 2016 at Detroit Medical Center (DMC), an acute-care academic health system located in Detroit, MI, USA was conducted. Adults $\geq 18$ years with a positive MRSA blood culture from infective endocarditis or pneumonia sources (as documented by the treating physician) and received $\geq 48 \mathrm{~h}$ of vancomycin initiated within $24 \mathrm{~h}$ of positive blood culture were included. Patients with multiple infection 
sources or who did not have an index MRSA isolate available for phenotypic testing were excluded. The institutional review boards of Wayne State University/DMC approved this study and granted waivers of informed consent. All procedures performed in studies involving human participants were in accordance with the ethical standards of the institutional and/or national research committee and with the 1964 Helsinki declaration and its later amendments or comparable ethical standards.

Index MRSA isolates from each bacteremia episode were screened and confirmed for hVISA via the modified PAP area under the curve (AUC) method [7]. The starting inoculum was approximately $1 \times 10^{9} \mathrm{cfu} / \mathrm{ml}$, adjusted to a $1 \times 10^{8} \mathrm{cfu} / \mathrm{ml}$ density and then spiral plated (Don Whitley Scientific, West Yorkshire, UK) onto brain heart infusion agar plates (Difco, Detroit, MI, USA). Each plate contained vancomycin at concentrations of $0,0.5,1,1.5,2,3$, 4,8 , or $16 \mathrm{mg} / \mathrm{l}$. Modified PAP values were compared to a reference Mu3 strain (ATCC 700689). An MRSA isolate was determined to be hVISA if the PAP-AUC to Mu3 ratio was $\geq 0.9$ [7]. The vancomycin PAP MIC was read as the lowest concentration to inhibit bacterial growth to below the detectable limit [10].

Clinical data were extracted from medical records by trained reviewers and entered into a structured data collection form within REDCap (Research Electronic Data Capture, Vanderbilt University, Nashville, TN, USA), an electronic data capture tool hosted at Wayne State University. The following variables were collected: demographics, past medical history and comorbid conditions, degree of comorbidity through the charlson comorbidity index [12] and severity of illness at time of positive MRSA blood culture through Acute Physiology and Chronic Health Evaluation (APACHE) II score [13], microbiologic data, treatment data, and clinical outcomes. The primary outcome was vancomycin composite failure, which comprised of persistent bacteremia $\geq 7$ days and 30-day mortality following index MRSA blood culture. Our institutional policy requires two sets of follow-up blood cultures obtained every $24-48 \mathrm{~h}$ for documentation of clearance, defined as negative blood cultures on two consecutive days.

Descriptive statistics, including baseline and clinical characteristics, were performed for the entire cohort. Bivariate associations between composite failure and potential predictors were conducted using the Pearson $X^{2}$ test or Fisher exact test for categorical data, and the Student's $t$ test or Mann-Whitney $U$ test for continuous data. Potential covariates identified in the bivariate analysis to be associated with the primary outcome at a $P$ value $<0.2$ level and were biologically-plausible were entered into the explanatory multivariable logistic regression model via a backward, stepwise approach to achieve a parsimonious model. Variables remained in the final model if they were significant at a $P$ value $<0.1$ level. Model fit was assessed through the Hosmer-Lemeshow goodness of fit test whereby models with a non-significant result were considered adequate. Classification and regression tree (CART) analysis was used to derive a significant PAP MIC breakpoint associated with the primary outcome. Statistical analysis was performed using SPSS Statistics, IBM SPSS Software, v.24.0 (IBM, Armonk, NY, USA).

\section{RESULTS}

A total of 191 patients with MRSA bacteremia were included. Among this cohort, 91 (47.6\%) and $100(52.4 \%)$ were from infective endocarditis and pneumonia sources, respectively. The mean age (SD) was $60.5( \pm 16.2)$ years, $64.4 \%$ male, and $79.1 \%$ African-American. The median Charlson Comorbidity Index was 3 (1, 5 ) and the most common comorbid conditions included diabetes mellitus (32.6\%), chronic kidney disease (30.9\%), and congestive heart failure $(25.1 \%)$. About one-third $(33 \%)$ of the patients were in the intensive care unit (ICU) during the index positive blood culture and the median (IQR) APACHE II score was $20(14,27)$. The majority (81\%) also received an infectious disease consult for the MRSA bacteremia. Nearly $20 \%$ of the isolates were hVISA. The median vancomycin PAP MIC was $3(2,3)$. Using CART analysis, $\mathrm{PAP} \mathrm{MIC} \geq 4 \mathrm{mg} / \mathrm{L}$ was associated with 


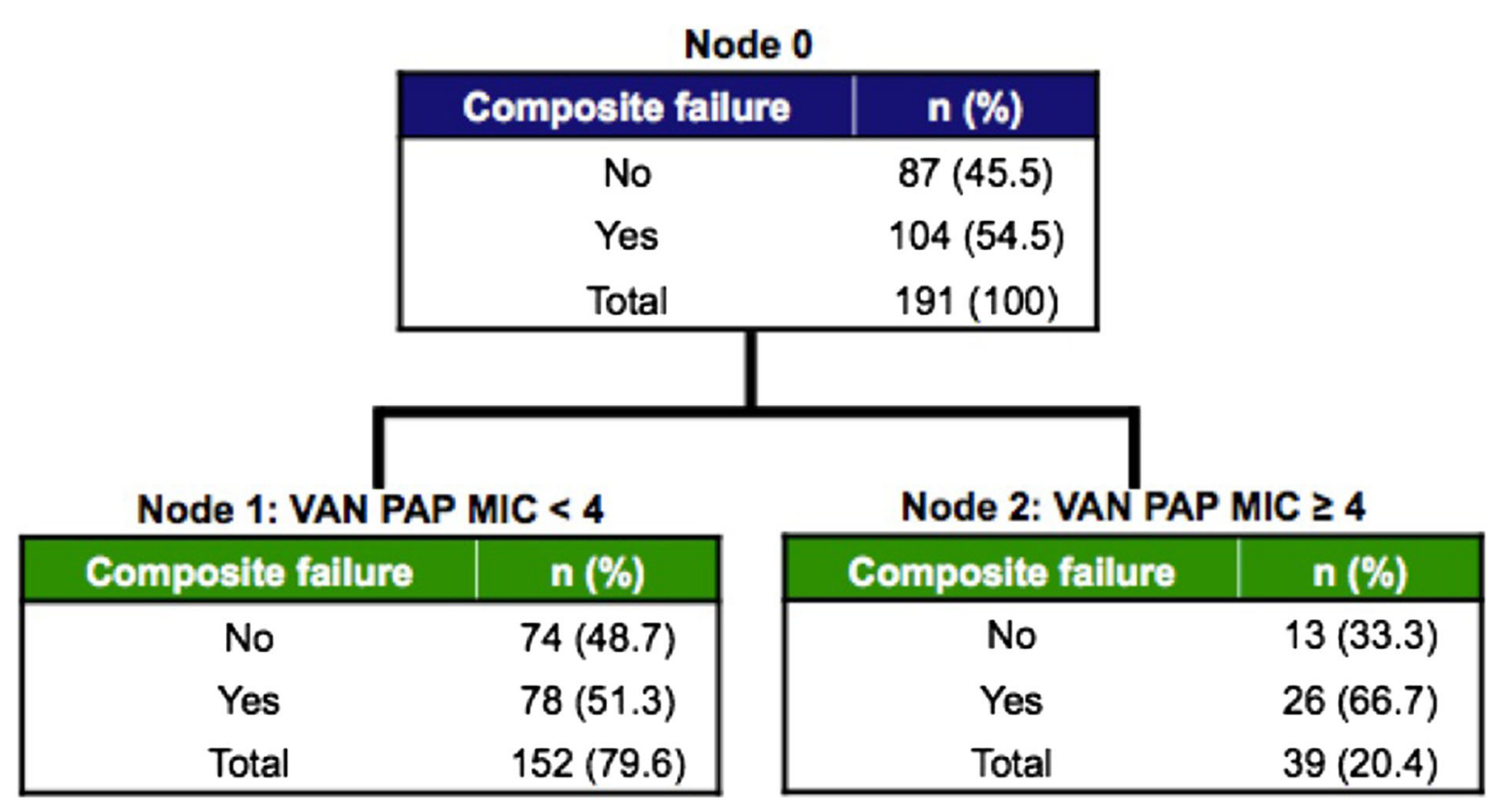

Fig. 1 Classification and regression tree (CART) analysis of VAN PAP MIC on composite failure

composite failure (Fig. 1). The overall median (IQR) length of hospital stay and bacteremia duration were $16(10,25)$ and $5(3,8)$ days, respectively. Within this cohort, 104 (54.5\%) experienced composite failure. Notable differences on bivariate analysis between patients who experienced composite failure versus those who did not are in Table 1.

Comparisons of the clinical outcomes between infective endocarditis and pneumonia are presented in Table 2 . A higher proportion of hVISA isolates were present in infective endocarditis than pneumonia ( 21 vs. $18 \%$ ). Patients with infective endocarditis also had significantly longer bacteremia durations ( 7 vs. 4 days, $p<0.001)$ and one additional day in length of stay (16 vs. 15 days, $p=0.029$ ). However, a higher proportion of pneumonia patients died at 30 days ( 37 vs. $22 \%, p=0.023$ ).

Candidate variables associated with composite failure that were entered into the multivariable logistic regression model included age, APACHE II score, African-American race, chronic hemodialysis, diabetes mellitus, hVISA phenotype, infection source, male sex, vancomycin treatment duration, and vancomycin $\mathrm{PAP}$ MIC $\geq 4 \mathrm{mg} / \mathrm{L}$. Results of the final model are presented in Table 3 (Hosmer-Lemeshow goodness-of-fit test $p=0.252$ ). When controlling for other covariates, vancomycin PAP MIC was not associated with composite failure. However, African-American race, infective endocarditis, high APACHE II score, older age, and shorter vancomycin treatment durations were significantly associated with composite failure. Male sex remained as an explanatory covariate in the final model $(p=0.10)$.

\section{DISCUSSION}

Vancomycin PAP MIC $\geq 4 \mathrm{mg} / \mathrm{L}$ has previously been shown to be associated with vancomycin treatment failures in MRSA infective endocarditis and remains a novel concept for this cohort [10]. Owing to the idea that higher vancomycin PAP MIC is collinear with the hVISA phenotype, this susceptibility method may be an alternative microbiological surrogate to predict outcomes in other high burden sources. However, the results from our study suggest that this breakpoint does not predict composite failure in MRSA pneumonia. There are noteworthy differences in outcomes 
Table 1 Clinical characteristics and outcomes of 191 patients with MRSA infective endocarditis and pneumonia

\begin{tabular}{|c|c|c|c|c|}
\hline Characteristic $n(\%)$ & $\begin{array}{l}\text { Overall cohort } \\
(n=191)\end{array}$ & $\begin{array}{l}\text { Failure } \\
(n=104)\end{array}$ & $\begin{array}{l}\text { Success } \\
(n=87)\end{array}$ & $P$ value \\
\hline \multicolumn{5}{|l|}{ Demographics } \\
\hline Age in years, mean $(\mathrm{SD})$ & $60.5(16.2)$ & $64.1(14.3)$ & $56.1(17.4)$ & 0 \\
\hline Male sex & $123(64.4)$ & $73(70.2)$ & $50(57.5)$ & 0.07 \\
\hline African-American & $151(79.1)$ & $92(88.5)$ & $59(67.8)$ & $<0.001$ \\
\hline Caucasian & $38(19.9)$ & $11(10.6)$ & $27(31)$ & $<0.001$ \\
\hline \multicolumn{5}{|l|}{ Comorbid conditions } \\
\hline $\begin{array}{l}\text { Charlson comorbidity index, median } \\
\text { (IQR) }\end{array}$ & $3(1,5)$ & $3(1,5)$ & $2(1,5)$ & 0.24 \\
\hline Diabetes mellitus & $62(32.6)$ & $38(36.5)$ & $24(27.6)$ & 0.19 \\
\hline Chronic pulmonary disease & $49(25.7)$ & $24(23.1)$ & $25(28.7)$ & 0.37 \\
\hline Congestive heart failure & $48(25.1)$ & $27(26)$ & $21(24.1)$ & 0.77 \\
\hline Cerebrovascular disease & $37(19.4)$ & $21(20.2)$ & $16(18.4)$ & 0.75 \\
\hline Chronic kidney disease & $59(30.9)$ & $34(32.7)$ & $25(28.7)$ & 0.56 \\
\hline Chronic dialysis & $26(13.6)$ & $19(18.3)$ & $7(8.0)$ & 0.04 \\
\hline Liver disease & $40(20.9)$ & $25(24)$ & $15(17.2)$ & 0.25 \\
\hline HIV & $8(4.2)$ & $1(1)$ & $7(8)$ & 0.02 \\
\hline Intravenous drug use & $50(26.2)$ & $29(27.9)$ & $21(24.1)$ & 0.56 \\
\hline Surgery in preceding 30 days & $6(3.1)$ & $1(1)$ & $5(5.7)$ & 0.09 \\
\hline $\begin{array}{l}\text { Hospitalization } \geq 48 \mathrm{~h} \text { in preceding } \\
90 \text { days }\end{array}$ & $74(38.7)$ & $41(39.4)$ & $33(37.9)$ & 0.83 \\
\hline MRSA infection in preceding year & $23(12)$ & $9(8.7)$ & $14(16.1)$ & 0.12 \\
\hline Antibiotics $\geq 24 \mathrm{~h}$ in preceding 90 days & $67(35.6)$ & $36(35)$ & $31(36.5)$ & 0.83 \\
\hline Infective endocarditis (IE) & $91(47.6)$ & $56(53.8)$ & $35(40.2)$ & 0.06 \\
\hline Definite IE & $70(76.1)$ & $42(73.7)$ & $28(80)$ & - \\
\hline Possible IE & $22(23.9)$ & $15(26.3)$ & $7(20)$ & - \\
\hline Tricuspid & $30(15.7)$ & $17(16.3)$ & $13(14.9)$ & - \\
\hline Mitral & $29(15.2)$ & $19(18.3)$ & $10(11.5)$ & - \\
\hline Aortic & $16(8.4)$ & $9(8.7)$ & $7(8)$ & - \\
\hline Unknown & $25(13.1)$ & $17(16.3)$ & $8(9.2)$ & - \\
\hline Native valve & $87(94.6)$ & $54(94.7)$ & $33(94.3)$ & - \\
\hline Prosthetic valve & $5(5.4)$ & $3(5.3)$ & $2(5.7)$ & - \\
\hline Pneumonia & $100(52.4)$ & $48(46.2)$ & $52(59.8)$ & 0.06 \\
\hline
\end{tabular}


Table 1 continued

\begin{tabular}{|c|c|c|c|c|}
\hline Characteristic $n$ (\%) & $\begin{array}{l}\text { Overall cohort } \\
(n=191)\end{array}$ & $\begin{array}{l}\text { Failure } \\
(n=104)\end{array}$ & $\begin{array}{l}\text { Success } \\
(n=87)\end{array}$ & $P$ value \\
\hline Community-acquired & $29(26.6)$ & $11(20.4)$ & $18(32.7)$ & - \\
\hline Healthcare-associated & $50(45.9)$ & $28(51.9)$ & $22(40)$ & - \\
\hline Hospital-acquired & $19(17.4)$ & $11(20.4)$ & $8(14.5)$ & - \\
\hline Ventilator-associated & $11(10.1)$ & $4(7.4)$ & $7(12.7)$ & - \\
\hline Infectious disease consult & $155(81.2)$ & $84(80.8)$ & $71(81.6)$ & 0.88 \\
\hline ICU at time of index culture & $63(33)$ & $36(34.6)$ & $27(31)$ & 0.6 \\
\hline APACHE II score, median (IQR) & $20(13.8,27.3)$ & $22(18,29)$ & $17(11,25)$ & $<0.001$ \\
\hline \multicolumn{5}{|l|}{ MRSA isolates } \\
\hline hVISA & $37(19.4)$ & $24(23.1)$ & $13(14.9)$ & 0.16 \\
\hline VAN PAP MIC, median (IQR) & $3(2,3)$ & $3(2,3.75)$ & $3(2,3)$ & 0.53 \\
\hline VAN PAP MIC $\geq 4 \mathrm{mg} / \mathrm{L}$ & $39(20.4)$ & $26(25)$ & $13(14.9)$ & 0.09 \\
\hline \multicolumn{5}{|l|}{ Vancomycin treatment } \\
\hline Duration in days, median (IQR) & $4(2,8)$ & $3(2,6)$ & $5(3,9)$ & 0 \\
\hline
\end{tabular}

$A P A C H E$ II acute physiology and chronic health evaluation II, HIV human immunodeficiency virus, $b V I S A$ heteroresistant vancomycin-intermediate $S$. aureus, $I C U$ intensive care unit, $I Q R$ interquartile range, $M I C$ minimum inhibitory concentration, $M R S A$ methicillin-resistant $S$. aureus, $P A P$ population analysis profile, $V A N$ vancomycin

Table 2 Clinical outcomes for patients with MRSA infective endocarditis versus pneumonia

\begin{tabular}{|c|c|c|c|c|}
\hline Characteristic $n$ (\%) & $\begin{array}{l}\text { Overall cohort } \\
(n=191)\end{array}$ & $\begin{array}{l}\text { Infective endocarditis } \\
(n=91)\end{array}$ & $\begin{array}{l}\text { Pneumonia } \\
(n=100)\end{array}$ & $P$ value \\
\hline \multicolumn{5}{|l|}{ MRSA isolates } \\
\hline hVISA & $37(19.4)$ & $19(20.9)$ & $18(18)$ & 0.62 \\
\hline VAN PAP MIC, median (IQR) & $3(2,3)$ & $3(2,3)$ & $3(3,3)$ & 0.01 \\
\hline \multicolumn{5}{|l|}{ Clinical outcomes } \\
\hline $\begin{array}{l}\text { Length of hospital stay in days, } \\
\text { median (IQR) }\end{array}$ & $16(10,25)$ & $16(11,25)$ & $15(7,23)$ & 0.03 \\
\hline Days of bacteremia, median (IQR) & $5(3,8)$ & $7(5,10)$ & $4(2,5)$ & $<0.001$ \\
\hline Bacteremia $\geq 7$ days & $65(34)$ & $47(51.6)$ & $18(18)$ & $<0.001$ \\
\hline 30-day mortality & $57(29.8)$ & $20(22)$ & $37(37)$ & 0.02 \\
\hline
\end{tabular}

hVISA heteroresistant vancomycin-intermediate $S$. aureus, $I Q R$ interquartile range, $M I C$ minimum inhibitory concentration, $M R S A$ methicillin-resistant $S$. aureus, $P A P$ population analysis profile, $V A N$ vancomycin 
Table 3 Multivariable logistic regression of predictors for vancomycin composite failure in MRSA infective endocarditis and pneumonia

\begin{tabular}{llll}
\hline Characteristic & OR (95\% CI) & AOR (95\% CI) & $\boldsymbol{P}$ value \\
\hline African-American & $3.64(1.72-7.71)$ & $4.22(1.77-10.1)$ & 0.001 \\
Infective endocarditis & $1.73(0.97-3.09)$ & $3.10(1.51-6.35)$ & 0.002 \\
Male & $1.74(0.96-3.17)$ & $1.81(0.90-3.63)$ & 0.097 \\
APACHE II score & $1.06(1.02-1.09)$ & $1.07(1.03-1.11)$ & 0.001 \\
Age & $1.03(1.01-1.05)$ & $1.03(1.00-1.05)$ & 0.028 \\
Vancomycin duration of treatment & $0.92(0.87-0.98)$ & $0.93(0.88-0.99)$ & 0.016 \\
Chronic dialysis & $2.56(1.02-6.40)$ & - & - \\
VAN PAP MIC $\geq 4 \mathrm{mg} / \mathrm{L}$ & $1.90(0.91-3.97)$ & - & - \\
hVISA phenotype & $1.71(0.81-3.60)$ & - & - \\
Diabetes mellitus & $1.51(0.82-2.80)$ & - & - \\
\hline
\end{tabular}

aOR adjusted odds ratio, $A P A C H E I I$ acute physiology and chronic health evaluation II, $b V I S A$ heteroresistant vancomycinintermediate $S$. aureus, $M I C$ minimum inhibitory concentration, $O R$ odds ratio, $P A P$ population analysis profile, VAN vancomycin

observed on bivariate analysis between the two infection types (Table 2).

As expected, infective endocarditis patients experienced prolonged bacteremia $\geq 7$ days and, consequently, longer lengths of hospital stay. This group also had a higher proportion of hVISA isolated with similar outcomes to those previously reported. Conversely, pneumonia patients experienced higher 30-day mortality rates. Considering that these outcomes are the two components of composite failure, it must be acknowledged that these competing outcomes limited our ability to detect a meaningful role of vancomycin PAP MIC for these two high burden sources.

Regression analysis of our cohort revealed that African-American race, infective endocarditis source, higher APACHE II scores, and older age should be considered when selecting empiric vancomycin treatment for serious MRSA infections. These characteristics are also supported by prior literature. Among our cohort, the median duration of vancomycin was 4 days with a significantly shorter duration observed between patients who experienced composite failure and those who did not (3 vs. 5 days, $p=0.002$ ). However, this observation provides limited information because we do not have patient-specific vancomycin pharmacokinetic parameters to firmly conclude appropriate treatment durations.

In patients with the aforementioned clinical characteristics associated with vancomycin failure, it may be reasonable to consider an alternative antibiotic especially in the critical first $48-72 \mathrm{~h}$. This may optimize treatment success in the presence of hVISA subpopulations that are not detected with contemporary susceptibility detection methods performed in clinical microbiology laboratories. As previously shown, vancomycin PAP MIC is a better predictor of hVISA than standard broth microdilution and Etest methods. Thus, the impetus for rapid and automated hVISA detection allows for prompt administration of active antibiotics and results in positive patient outcomes.

This study included a substantial cohort of two high burden MRSA infection types: infective endocarditis and pneumonia. Owing to its retrospective, single-center design, there are several limitations to note. Firstly, this study was performed at one institution with specific practice patterns, which may limit the applicability of these findings to other sites. Secondly, the possibility for misclassification of the vancomycin PAP MIC breakpoint must be 
considered. Although the index MRSA isolate was tested for hVISA, the retrospective nature limits our ability to reliably discern whether this truly represented the first bacteremia episode versus subsequent recurrences and treatment failures from prior vancomycin exposures. Thirdly, all isolates were from the blood and this may not accurately reflect the subpopulations found at the primary site of infection (i.e., heart valves and lungs). It may be of interest to explore the relationship between vancomycin PAP MIC from MRSA respiratory cultures and its impact on outcomes in pneumonia. Lastly, our study did not include full molecular typing of isolates, thus it is not possible to comment on the role of clonal relatedness on outcomes.

\section{CONCLUSION}

In conclusion, our study suggests that vancomycin PAP MIC may have a specific role in predicting prolonged bacteremia durations in high inoculum infections with known persistence (e.g., infective endocarditis). However, this alternative MIC detection method may be less applicable to predict outcomes in MRSA bacteremic pneumonia. It is likely that pneumonia patients experience higher mortality rates, thereby effectively limiting their ability to experience bacteremia persistence. Until there is rapid diagnostic technology to efficiently detect hVISA, it may be more reasonable to direct empirical treatment for severe MRSA infections based on African-American race, infective endocarditis source, higher APACHE II scores, and older age. It would also be noteworthy for future studies to examine the role of heteroresistance and PAP MIC on treatment outcomes from other lipopeptide antibiotics (e.g., daptomycin) for MRSA bacteremia.

\section{ACKNOWLEDGEMENTS}

Funding. No funding or sponsorship was received for this study or publication of this article.
Authorship. All named authors meet the International Committee of Medical Journal Editors (ICMJE) criteria for authorship for this article, take responsibility for the integrity of the work as a whole, and have given their approval for this version to be published. Assistance with data collection was provided by Dana El Masri, Pharm.D.

Disclosures. Trang D. Trinh, Evan J. Zasowski, Kimberly C. Claeys, Anthony M. Casapao, Matthew Compton, Abdalhamid Lagnf, Shravya D. Kidambi, Donald P. Levine, and Michael J. Rybak have nothing to disclose.

Compliance with Ethics Guidelines. All procedures performed in studies involving human participants were in accordance with the ethical standards of the institutional and/or national research committee and with the 1964 Helsinki declaration and its later amendments or comparable ethical standards. Informed consent was not obtained because of the retrospective nature of this study.

Data Availability. The datasets during and/ or analyzed during the current study are available from the corresponding author on reasonable request.

Open Access. This article is distributed under the terms of the Creative Commons Attribution-NonCommercial 4.0 International License (http://creativecommons.org/licenses/ by-nc/4.0/), which permits any noncommercial use, distribution, and reproduction in any medium, provided you give appropriate credit to the original author(s) and the source, provide a link to the Creative Commons license, and indicate if changes were made.

\section{REFERENCES}

1. David MZ, Medvedev S, Hohmann SF, Ewigman B, Daum RS. Increasing burden of methicillin-resistant Staphylococcus aureus hospitalizations at US academic medical centers, 2003-2008. Infect Control Hosp Epidemiol. 2012;8:782-9. 
2. Dantes R, Mu Y, Belflower R, et al. National burden of invasive methicillin-resistant Staphylococcus aureus infections, United States, 2011. JAMA Intern Med. 2013;21:1970-8.

3. Pfuntner A, Wier LM, Steiner C. Costs for hospital stays in the United States, 2011: Statistical brief \#168. In: Healthcare cost and utilization project (HCUP) statistical briefs. Rockville (MD): 2006.

4. Xu J, Murphy SL, Kochanek KD, Bastian BA. Deaths: final data for 2013. Natl Vital Stat Rep. 2016;2:1-119.

5. Liu C, Bayer A, Cosgrove SE, et al. Clinical practice guidelines by the infectious diseases society of america for the treatment of methicillin-resistant Staphylococcus aureus infections in adults and children. Clin Infect Dis. 2011;3:e18-55.

6. van Hal SJ, Paterson DL. Systematic review and meta-analysis of the significance of heterogeneous vancomycin-intermediate Staphylococcus aureus isolates. Antimicrob Agents Chemother. 2011;1:405-10.

7. Wootton M, Howe RA, Hillman R, Walsh TR, Bennett PM, MacGowan AP. A modified population analysis profile (PAP) method to detect hetero-resistance to vancomycin in Staphylococcus aureus in a UK hospital. J Antimicrob Chemother. 2001;4:399-403.

8. Musta AC, Riederer K, Shemes S, et al. Vancomycin MIC plus heteroresistance and outcome of methicillin-resistant Staphylococcus aureus bacteremia: trends over 11 years. J Clin Microbiol. 2009;6:1640-4.

9. Bae IG, Federspiel JJ, Miro JM, et al. Heterogeneous vancomycin-intermediate susceptibility phenotype in bloodstream methicillin-resistant Staphylococcus aureus isolates from an international cohort of patients with infective endocarditis: prevalence, genotype, and clinical significance. J Infect Dis. 2009;9:1355-66.

10. Casapao AM, Davis SL, McRoberts JP, et al. Evaluation of vancomycin population susceptibility analysis profile as a predictor of outcomes for patients with infective endocarditis due to methicillin-resistant Staphylococcus aureus. Antimicrob Agents Chemother. 2014;8:4636-41.

11. Harigaya Y, Bulitta JB, Forrest A, et al. Pharmacodynamics of vancomycin at simulated epithelial lining fluid concentrations against methicillin-resistant Staphylococcus aureus (MRSA): implications for dosing in MRSA pneumonia. Antimicrob Agents Chemother. 2009;9:3894-901.

12. Charlson ME, Pompei P, Ales KL, MacKenzie CR. A new method of classifying prognostic comorbidity in longitudinal studies: development and validation. J Chronic Dis. 1987;5:373-83.

13. Knaus WA, Draper EA, Wagner DP, Zimmerman JE. APACHE II: a severity of disease classification system. Crit Care Med. 1985;10:818-29. 